Rev. Elev. Méd. vét. Pays trop., 1971, 24 (3) : 373-79

\title{
Étude sur l'allergie, les anticorps précipitants et les anticorps fixant le complément au cours de l'infestation des bovins par Fasciola gigantica
}

\author{
par J. BLANCOU $(*)$, A. BOUCHET $(*)$ et P. DAYNES $(*)$
}

\begin{abstract}
RESUME
Trois épreuves (intra-dermo réaction, fixation du complément, double diffusion en gélose) ont été réalisées chez 209 zébus infestés naturellement ou artificiellement par Fasciola gigantica.

L'intra-dermo réaction révèle 60 à 70 p. 100 des sujets infestés (mais avec 30 à 35 p. 100 de résultats par excès), la fixation du complément $23-48$ p. 100 ( 8 p. 100 de résultats par excès) et la diffusion en gélose 16 à 36 p. 100 (sans résultat par excès).
\end{abstract}

\section{INTRODUCTION}

La fasciolose, très répandue dans le monde entier, semblait jusqu'à ces dernières années avoir épargné Madagascar.

En 1966, l'un de nous attirait l'attention sur un premier foyer de distomatose à Fasciola gigantica, décrivait l'hôte intermédiaire Limnaea natalensis et reproduisait expérimentalement le cycle biologique du parasite (7).

Depuis quatre ans, la maladie s'est considérablement étendue à travers la Grande Ile, amenant le Service d'Helminthologie du Laboratoire Central de l'Elevage à étudier tous les moyens de diagnostic et de prophylaxie qui lui soient applicables.

Il est apparu très utile d'explorer dans ce but des méthodes immunologiques destinées à contrôler les réactions du zébu malgache à cette nouvelle parasitose et à apprécier ses réactions au cours de traitements expérimentaux ou d'immunisation ultérieure, naturelle ou artificielle. Ces méthodes, largement appliquées dans l'infestation à Fasciola hepatica ont été très peu

(") I.E.M.V.T., Laboratoire Central de l'Elevage, Tananarive. étudiées dans le cas de Fasciola gigantica, méritant à ce titre d'être éprouvées avant la méthode plus récente de l'immunofluorescence indirecte.

Ce sont les résultats de nos essais qui font l'objet de la présente étude.

\section{MATERIEL ET METHODES}

\section{ANIMAUX D'EXPERIENCE}

Au total 209 zébus ont été testés. Il n'a malheureusement pas été possible d'obtenir dans tous les cas des sujets sûrement indemnes dautres helminthoses et en particulier de trématodoses (Paramphistomidae, Gastrothylacidae, Eurythrema) rencontrés souvent à Madagascar (8).

\section{A. Infestation naturelle}

Les animaux infestés naturellement, utilisés dans cet essai, provenaient du Moyen-Ouest de Madagascar; seuls ont été retenus ceux qui présentaient, parmi divers oufs d'autres parasites, ceux de Fasciola gigantica. Le bilan total de leur parasitisme n'a pu être établi, car seul 
l'examen coprologique était réalisable sur ces bovins appartenant à des particuliers.

\section{B. Infestation expérimentale}

Quarante sujets en provenance d'une région indemne de fasciolose, ont été infestés chacun avec 800 métacercaires. L'infestation a réussi dans tous les cas. A l'autopsie, ces animaux étaient porteurs de Fasciola gigantica, mais aussi de Paramphistomum sp. et de Sétaires, Setaria labiatopapillosa. Certains de ces animaux ont reçu un traitement par un douvicide nouveau, le Rafoxanide; les résultats de cet essai font l'objet d'une étude complète qui sera publiée ultérieurement.

\section{Témoins}

Soixante sérums témoins provenaient de bovins abattus à l'abattoir de Tananarive et reconnus indemnes de fasciolose. Le bilan exact de l'infestation par d'autres helminthes n'a pas été réalisable.

Douze sujets témoins ont été placés dans les mêmes conditions d'habitat et de nourriture que les quarante animaux infestés artificiellement. Ces animaux qui provenaient d'une région indemne de fasciolose, ont été contrôlés pendant un mois du point de vue coproscopique; à l'autopsie, ces animaux se sont révélés infestés par Fasciola gigantica, Paramphistomum sp. et Setaria labiatopapillosa.

\section{TECHNIQUES D'ETUDE}

Trois techniques ont été utilisées : Précipitation en gélose (méthode Oudin-Ouchterlony). Fixation du complément et recherche de l'état d'hypersensibilité par injection intradermique.

\section{A. Matériel}

a) Sérums : le sang des bovins est recueilli, par ponction veineuse de la jugulaire, en tube à essais. Le sérum, prélevé après la rétraction du caillot (à température ambiante) est conservé à moins $20^{\circ} \mathrm{C}$ jusqu'à son analyse.

b) Antigène: nous n'avons utilisé que des antigènes bruts ou délipidés, préparés selon đes méthodes inspirées de celle décrite par R. DESCHIENS (9) et R. PAUTRIZEL (15).

\section{Antigène destiné à la réaction de précipitation en gélose}

Les douves sont récoltées vivantes dans les canaux biliaires aussitôt après l'abattage, lavées dans 5 bains successifs d'eau physiologique stérile à $4^{\circ} \mathrm{C}$, triées, de façon à ne conserver que les spécimens en bon état, puis broyées aussitôt dans un appareil de type Turmix prérefroidi au congélateur. Ce broyage est effectué en présence d'un égal volume d'eau physiologique froide. Lorsque le mélange est devenu homogène, il subit un cycle de 10 congélations (à moins $20^{\circ} \mathrm{C}$ ) puis décongélations brutales (à $37^{\circ} \mathrm{C}$ ) avant d'être réparti en ampoules de $2 \mathrm{ml}$ qui seront conservées à moins $20^{\circ} \mathrm{C}$ ou lyophilisées.

\section{Antigène destiné à la réaction de fixation du complément}

Le broyat précédent est additionné d'eau physiologique de façon à obtenir une suspension finale contenant $1 / 10$ de vers en poids sec. En pratique, les vers étant pesés humides après essorage, nous rajoutons 100 grammes de vers à $140 \mathrm{ml}$ d'eau physiologique. Le broyat subit alors le cycle de congélation - décongélation et les débris solides sont éliminés par une centrifugation de 15 minutes à 2.000 tours par minute. Le surnageant est distribué en ampoules de $2 \mathrm{ml}$ conservées à moins $20^{\circ} \mathrm{C}$ ou lyophilisées.

\section{Antigène destiné \\ à la réaction intra-dermique}

Nous avons utilisé deux antigènes différents pour les injections intra-dermiques :

a) l'antigène de la fixation du complément décrit précédemment, après dilution à 10 p. 100.

b) un antigène délipidé préparé selon la méthode décrite par R. PAUTRIZEL (15); le cryolysat de Douves est traité par 5 fois son volume d'alcool absolu, puis d'éther, desséché sous vide et soumis à une extraction en eau distillée.

Nous avons dosé l'azote total de cet antigène et utilisé une dilution contenant 20 microgrammes d'azote par $\mathrm{ml}$.

\section{B. Exécution des réactions}

a) Précipitation en gélose

La réaction est effectuée en boîte de Petri 
où sont coulés $15 \mathrm{ml}$ d'une gélose de composition suivante:

- Agar Noble (DIFCO) . . . 6 g

- Merthiolate . . . . . . $0,1 \mathrm{~g}$

— Tampon P.B.S. . . . . . $700 \mathrm{ml}$

La réaction se produit, à température ordinaire, en 12 à 48 heures. Après ce délai, les boîtes sont placées à plus $4^{\circ} \mathrm{C}$ pour éviter les contaminations et la lecture définitive est effectuée après 6 jours.

Une réaction positive se traduit par l'apparition d'un ou plusieurs traits de précipitation, blancs, très nets, entre deux puits. On peut s'assurer de la spécificité du précipité antigèneanticorps par un lavage dans une solution de citrate trisodique ou un lavage prolongé en eau physiologique.

\section{b) Fixation du complément}

Nous utilisons la technique de Kolmer adaptée par R. DESCHIENS (9).

La réaction se lit après une heure à $37^{\circ} \mathrm{C}$. Nous ayons observé assez souvent des " phéno- mènes de zone " et d'anticomplémentarité aux faibles dilutions des sérums, ce qui complique parfois la lecture.

\section{c) Recherche de l'état d'hypersensibilité}

L'état d'allergie a été recherché par la méthode des injections intra-dermiques d'antigène sur des bovins infestés artificiellement 15 semaines auparavant. Nous avons injecté simultanément à chaque bovin les deux antigènes précédemment décrits.

1. L'antigène délipidé est injecté dans le derme du pli sous-caudal, avec une seringue à tuberculination, sous le volume de $0,2 \mathrm{ml}$.

2. L'antigène non délipidé, qui est l'antigène de la fixation du complément (fraîchement préparé), est injecté dans le derme du plat de l'encolure, sans rasage préalable, sous le volume de $0,2 \mathrm{ml}$, avec une seconde seringue à tuberculination.

La réaction est appréciée par le diamètre de la zone réactionnelle (odème) noté de la $15^{\mathrm{e}}$ à $1 \mathrm{a} 90^{\mathrm{e}}$ minute après linjection.

\section{RESULTATS}

Les résultats obtenus sont exprimés sous forme de tableaux :

\section{ANIMAUX NATURELLEMENT INFESTES}

TABLEAU $\mathrm{N}^{\circ} \mathrm{I}$

Animaux nature1lement infestês

\begin{tabular}{|c|c|c|c|c|c|c|}
\hline \multirow{3}{*}{$\begin{array}{c}\text { Précipitation } \\
\text { en } \\
\text { gélose }\end{array}$} & \multicolumn{6}{|c|}{ Rés s u l tats } \\
\hline & $\begin{array}{l}\text { Bovins sûrement infestés } \\
\text { (êliminant des aeufs de } \\
\text { Fasciola gigantica) }\end{array}$ & & \multicolumn{4}{|c|}{$\begin{array}{l}20 \text { précipitations spécifiques sur } \\
79 \text { sérums examinês }\end{array}$} \\
\hline & $\begin{array}{l}\text { Bovins tëmoins non } \\
\text { infestës }\end{array}$ & \multicolumn{5}{|c|}{$\begin{array}{l}\text { Aucune précipitatıon spécifique } \\
\text { sur } 60 \text { sérums analysés. }\end{array}$} \\
\hline \multirow{4}{*}{ Fixation } & \multirow{3}{*}{$\begin{array}{l}\text { Bovins sûrement infestés } \\
\text { (éliminant des oeufs de } \\
\text { Fasciola gigantica) }\end{array}$} & \multicolumn{5}{|c|}{$D \perp l u t i \circ n$ d u sé $r u m$} \\
\hline & & Pur & $1 / 2$ & $1 / 4$ & $1 / 8$ & $1 / 16$ \\
\hline & & $\frac{7}{5}$ & $6 \frac{3}{5}$ & $6 \frac{9}{5}$ & $6 \frac{8}{5}$ & $6 \frac{5}{5}$ \\
\hline & \multirow{3}{*}{$\begin{array}{l}\text { Bovins témoins non } \\
\text { infestés }\end{array}$} & \multicolumn{5}{|c|}{ Di l u t ion d u sêrum } \\
\hline \multirow{2}{*}{ complëment } & & Pur & $1 / 2$ & $1 / 4$ & $1 / 8$ & $1 / 16$ \\
\hline & & $\frac{18}{44}$ & $\frac{2}{44}$ & $\frac{0}{44}$ & $\frac{2}{44}$ & $\frac{0}{44}$ \\
\hline
\end{tabular}




\section{ANIMAUX INFESTES ARTIFICIELLEMENT}

\section{A. Précipitation en gélose}

Aucun sérum d'animal infesté n'a présenté d'anticorps précipitant entre le $1^{\mathrm{er}}$ et le $150^{\mathrm{e}}$ jour suivant l'infestation (analyse mensuelle).

\section{B. Fixation du complément}

Cette méthode a été utilisée sur trois lots de bovins : infestés, infestés puis traités, non infestés. Les résultats sont résumés dans le tableau suivant :

TABLEAU $N^{\circ} I I$

\begin{tabular}{|c|c|c|c|c|c|}
\hline \multicolumn{6}{|c|}{ 1-Lot de 28 animaux infestës non traités } \\
\hline $\begin{array}{c}\text { Titre de la fixation } \\
\text { du complëment }\end{array}$ & \multicolumn{5}{|c|}{ Nombre de sujets présentant ce titre } \\
\hline & $\begin{array}{c}\text { Avant } \\
\text { infestation }\end{array}$ & $\begin{array}{l}3 \text { semaines } \\
\text { après }\end{array}$ & $\begin{array}{l}7 \text { semaines } \\
\text { après }\end{array}$ & $\begin{array}{l}11 \text { semaines } \\
\text { après }\end{array}$ & $\begin{array}{l}15 \text { semaines } \\
\text { après }\end{array}$ \\
\hline Absence de fixation & 16 sur 27 & 1 sur 27 & 2 sur 27 & 1 sur 24 & Aucun \\
\hline $\begin{array}{l}\text { Fixation par sérum } \\
\text { pur }\end{array}$ & 9 sur 27 & 6 sur 27 & Aucun & 2 sur 24 & 4 sur 28 \\
\hline $\begin{array}{l}\text { Fixation pax sêrum } \\
\text { dilué ã } 1 / 2\end{array}$ & 2 sur 27 & 2 sur 27 & 1 sur 27 & 1 sur 24 & 3 sur 28 \\
\hline $\begin{array}{l}\text { Fixation par sérum } \\
\text { dilué à } 1 / 4\end{array}$ & Aucur & 5 sur 27 & 5 sur 27 & 4 sur 24 & 1 sur 28 \\
\hline $\begin{array}{l}\text { Fixation par sêrum } \\
\text { dilué à } 1 / 8\end{array}$ & Aucun & 13 sur 27 & 4 sur 27 & 4 sur 24 & 7 sur 28 \\
\hline $\begin{array}{l}\text { Fixation par sérum } \\
\text { diluê à } 1 / 16\end{array}$ & Aucun & Aucun & 15 sur 27 & 12 sur 24 & 13 sur 28 \\
\hline \multicolumn{6}{|c|}{$\begin{array}{c}\text { 2-Lot de } 10 \text { animaux infestés et traitës huit semaines plus tard } \\
\text { (Sêrums étudiés aux mêmes dates) }\end{array}$} \\
\hline Absence de Eixation & 5 sur 10 & Aucun & 1 sur 10 & Aucun & Aucun \\
\hline $\begin{array}{l}\text { Fixation par sërum } \\
\text { pur }\end{array}$ & 4 sur 10 & Aucun & Aucun & $1 \operatorname{sur} 7$ & Aucun \\
\hline $\begin{array}{l}\text { Fixation par sêrum } \\
\text { dilué à } 1 / 2\end{array}$ & 1 sur 10 & Aucun & 1 sur 10 & Aucun & 1 sur 10 \\
\hline $\begin{array}{l}\text { Fixation par sérum } \\
\text { dilué à } 1 / 4\end{array}$ & Aucun & 2 sur 10 & 1 sur 10 & 1 sur 7 & 1 sur 10 \\
\hline $\begin{array}{l}\text { Fixation par sêrum } \\
\text { dilué à } 1 / 8\end{array}$ & Aucun & 8 sur 10 & 4 sur 10 & 1 sur 7 & 2 sur 10 \\
\hline $\begin{array}{l}\text { Fixation par sērum } \\
\text { diluê à } 1 / 16\end{array}$ & Aucun & Aucun & 3 sur 10 & 4 sur 7 & 6 sur 10 \\
\hline \multicolumn{6}{|c|}{ 3-Lot de 12 animaux non infestés : sérums étudiês aux mêmes dates } \\
\hline Absence de Fixation & $3 \operatorname{sur} 7$ & $2 \operatorname{sur} 7$ & Aucun & 2 sur 10 & 1 sur 12 \\
\hline $\begin{array}{l}\text { Fixation par sërum } \\
\text { pur }\end{array}$ & $3 \operatorname{sur} 7$ & 3 sux 7 & 3 sur 10 & 2 sur 10 & 5 sur 12 \\
\hline $\begin{array}{l}\text { Fixation pax sérum } \\
\text { dilue à } 1 / 2\end{array}$ & Aucun & $1 \operatorname{sur} 7$ & 3 sur 10 & 2 sur 10 & 2 sur 12 \\
\hline $\begin{array}{l}\text { Fixatıon par sérum } \\
\text { dilué à } 1 / 4\end{array}$ & Aucun & Aucun & Aucun & 1 sur 10 & 1 sur 12 \\
\hline $\begin{array}{l}\text { Ejxation par sérum } \\
\text { dilué à } 1 / 8\end{array}$ & 1 sur 7 & $1 \operatorname{sur} 7$ & Aucun & Aucun & 1 sur 12 \\
\hline $\begin{array}{l}\text { Fixation par sérum } \\
\text { dilué à } 1 / 16\end{array}$ & Aucun & Aucun & 4 sur 10 & 3 sur 10 & 2 sur 12 \\
\hline
\end{tabular}




\section{Intra-dermo réaction}

La réaction a été recherchée sur les trois lots précédents, le point d'injection de l'antigène étant observé toutes les 20 minutes, de la $15^{\text {e }}$ à la $90^{\mathrm{e}}$ minute. Le diamètre de la zone d'infiltration réactionnelle a été mesuré au pied à coulisse : il variait de 1 à $25 \mathrm{~mm}$. Le plus grand nombre de réactions a été observé entre la $40^{\mathrm{e}}$ et la $70^{\mathrm{e}}$ minute. Si l'on ne prend en considération que les réactions d'un diamètre supérieur à $5 \mathrm{~mm}$, le résultat global des intra-dermo réactions peut être résumé dans le tableau cidessous :

TABLEAU $N^{\circ}$ III

Sombre de réactıons d'un diamètre supérieur à 5 mm en fonction du moment de la lecture et de l'antigène injecté.

\begin{tabular}{|c|c|c|c|c|}
\hline \multirow[b]{2}{*}{ Lot de bovins } & \multicolumn{2}{|c|}{$\begin{array}{c}\text { Réaction lue entre la 40ème et } \\
\text { la 50ême minute }\end{array}$} & \multicolumn{2}{|c|}{$\begin{array}{l}\text { Réaction lue entre la 60ème et } \\
\text { 1a 70ême minute }\end{array}$} \\
\hline & $\begin{array}{l}\text { Antigène non } \\
\text { délipidé } \\
\text { (encolure) }\end{array}$ & $\begin{array}{c}\text { Antigẽne } \\
\text { délipidé } \\
\text { (p1i sous-caudal) }\end{array}$ & $\begin{array}{l}\text { Antigène non } \\
\text { délipidé } \\
\text { (encolure) }\end{array}$ & $\begin{array}{ll} & \text { Antıgène } \\
& \text { délipidé } \\
\text { (pli sous-caudal) }\end{array}$ \\
\hline Témolns & 2 sur 12 & 4 sur 12 & $2 \operatorname{sur} 12$ & 2 sur 12 \\
\hline $\begin{array}{l}\text { Infestés } \\
\text { non traités }\end{array}$ & 9 sur 19 & 13 sur 19 & 11 sur 19 & $12 \operatorname{sur} 19$ \\
\hline $\begin{array}{l}\text { Infestés } \\
\text { traités }\end{array}$ & 9 sur 10 & 8 sur 10 & 8 sur 10 & 7 sur 10 \\
\hline
\end{tabular}

\section{DISCUSSION}

Nous examinerons successivement la valeur des trois méthodes étudiées dans le cas d'infestation, naturelle ou artificielle, par Fasciola gigantica et avec les méthodes et le matériel antigénique décrits précédemment.

\section{Méthode de précipitation en gélose}

En cas d'infestation artificielle: la méthode n'a pu détecter d'anticorps, même 15 semaines après l'infestation. Ceci s'explique par le taux élevé d'anticorps nécessaire à l'apparition de la réaction et diffère donc de ce qu'ont observé A. CAPRON (6) et J.J. SINCLAIR (17) chez le lapin ou M. BABENSKAS (1) chez les bovins infestés par Fasciola hepatica.

En cas d'infestation naturelle: la méthode a détecté 20 sujets sur 79 bovins infestés soit 16 à 36 p. 100 des cas (intervalle de confiance apprécié au risque 5 p. 100) sans jamais donner de résultats par excès. Ceci est en accord avec les résultats de FRICK (11) mais inférieur au taux rapporté par M.A. BABYANSKAS (2) dans l'infestation par Fasciola hepatica.

En conclusion: la facilité d'exécution de la réaction et la certitude du diagnostic, en cas de réponse positive, en font, dans certains cas, une méthode de valeur.

\section{Méthode de fixation du complément}

En cas d'infestation artificielle: les anticorps apparaissent très tôt. Leur titre est ensuite assez variable, comme le note A. CAPRON (6) chez le lapin, tout en restant assez élevé au moins jusqu'à la $15^{\mathrm{e}}$ semaine suivant l'infestation.

Malheureusement, la méthode perd de sa valeur du fait de l'existence de réactions faussement positives, déjà observées par J. BENEX (3) et P.B. MATHUR (13) chez le mouton infesté par Fasciola hepatica.

En cas d'infestation naturelle : le taux d'anticorps est très variable selon les individus, sans doute du fait de la différence d'ancienneté et d'intensité des infestations.

En conclusion: Avec la méthode et les antigènes que nous avons utilisés, si nous admettons comme significatifs les taux supérieurs à la dilution $1 / 2$, il serait possible de détecter 23 à 48 p. 100 des animaux infestés naturellement. Mais cette méthode donne 8 p. 100 de résultats par excès ce qui lui enlève beaucoup de sa valeur. Par ailleurs l'exécution de la réaction reste délicate et longue.

\section{Méthode de l'intra-dermo-réaction}

En cas d'infestation artificielle: Si l'on 
admet comme positives les réactions égales ou supérieures à $5 \mathrm{~mm}$, il serait possible de détecter par l'intra-dermo-réaction de 60 à 70 p. 100 des animaux infestés, l'intra-dermo-réaction au pli sous caudal avec l'antigène délipidé paraissant plus sensible. Après traitement des animaux infestés, la proportion de réactions positives atteint 80 à 90 p. 100 , et c'est alors l'intradermo-réaction à l'encolure avec l'antigène non délipidé qui semble la plus sensible. L'hypersensibilité augmenterait notablement après traitement, vraisemblablement du fait de la lyse des parasites. On note cependant des réactions positives en excès pouvant aller jusqu'à 30 à 35 p. 100 des cas.

Les proportions de réactions positives apparaissent plus élevées si l'on combine les résultats obtenus avec les deux méthodes et les deux antigènes utilisés, ou si l'on combine les résultats obtenus aux deux périodes considérées. On atteint le pourcentage maximal en combinant les quatre paramètres, mais les résultats par excès suivent la même progression.

Ces résultats peuvent être rapprochés de ceux obtenus en cas d'infestation par Fasciola hepatica où la proportion de résultats positifs, en cas d'infestation confirmée, est très différente selon les auteurs; elle varie de 50 à 100 p. 100 selon M. BABENSKAS (1), P. CABRAL GONCALVES (5), V. FAVATTI (10) W. FRICK (11), T.M. TOPACIO (19), J. VAREJCKO (20). Elle est de 100 p. 100 en cas d'infestation par Fasciola gigantica selon B. PATNAIK (14).

\section{CONCLUSION}

Les méthodes de la fixation du complément et de l'intra-dermo-réaction peuvent compléter utilement le diagnostic d'infestation par Fasciola gigantica lorsque l'examen coproscopique est encore négatif, c'est-à-dire en début d'infestation.

Lors d'infestations anciennes, la méthode de précipitation en gélose peut confirmer les résultats coproscopiques ou permettre d'assurer des enquêtes sur le parasitisme à partir de collection de sérums.

En ce qui concerne les deux premières méthodes, on notera que les réactions faussement positives sont fréquentes alors que la coproscopie, qui donne des résultats plus tardifs, fournit souvent des résultats faussement négatifs.

Ceci est un phénomène fréquent en immunologie, particulièrement net dans l'étude des parasites constitués par une "mosaïque d'antigène " qui entraîne des réactions de groupe. A Madagascar, l'infestation concomitante par les helminthes du groupe des Trématodes suffirait à expliquer les fausses réactions observées : les relations immunitaires probables entre ces diverses trématodoses nécessiteraient, à elles seules, une étude spéciale complète.

\section{SUMMARY}

Study of intra-dermal, complement fixation and precipitin tests in cattle infected with Fasciola gigantica

Three tests (intra-dermal, complement fixation, double diffusion in agar) were performed in 209 cattle naturally or experimentally infected with Fasciola gigantica.

Intra-dermal test detected $60-70$ p. 100 of infected animals (but 30-35 p. 100 false positive results) complement fixation 23-48 p. 100 ( 8 p. 100 false positive) and precipitin test $16-36$ p. 100 (no false positive results).

\section{RESUMEN}

Estudio sobre la alergia, los anticuerpos precipitantes y los anticuerpos fijando el complemento durante la infestación de los bovinos por Fasciola gigantica

Se realizaron tres pruebas (intradermoreacción, fijación del complemento, doble difusión en gelosa) en 209 cebues naturalmente o artificialmente infestados por Fasciola gigantica.

La intradermoreacción revela 60 a 70 p. 100 de los animales infestados (pero con 30 a 35 p. 100 de resultados por exceso), la fijación del complemento 23 a 48 p. 100 ( 8 p. 100 de resultatos por exceso) y la difusión en gelosa 16 a 36 p. 100 (sin resultado por exceso). 


\section{BIBLIOGRAPHIE}

1. BABENSKAS (M.), "Immunological diagnosis of bovine fascioliasis", Proc. 18th World Vet. Congr. Paris, 1967, 1 : 131-34.

2. BABYANSKAS (M. A.). * Immunobiological reactions for diagnosis of fascioliasis in farm animals ", Proc. Conf. All-Union Soc. HelminthMoscow, déc. 1962, Part II : 15-16.

3. BENEX (J.). LAMY (L.), GLEDEL (J.). «Etude de la réaction de fixation du complément à l'antigène distomien chez le mouton $», B u l l$. Soc. Path. exot., 1959, 52 (1): 83-87.

4. BIGUET (J.), CAPRON (A.), TRAN VAN KY (P.), «Les antigènes de Fasciola hepatica, étude électrophorétique et immunoélectrophorétıque. Identufication des fractions et comparaison avec les antigènes correspondant à sept autres helminthes ", Ann. Parasit. hum. comp. 1962, 37 (3) : 221

5. CABRAL GONCALVES (P), LOPES SALES (R.), «Practical value of the skin test for Fasciola in cattle », Rev Fac. agron vet., Porto Alegre 1964: 41-50.

6. CAPRON (A.) et collab., "Apport de la distomatose expérimentale à la connaissance de la distomatose humaine à Fasciola he patica. Aspects immunologiques », Rev. Imminol., Paris, 1965, 29: $25-42$.

7. DAYNES (P.), « Note préliminaire sur la présence de Fasciola gigantica à Madagascar », Rev. Elev. Méd. vét. Pays trop. 1967, 20 (4): 557-62.

8. DAYNES (P.), \& Note sur les helminthoses des animaux domestiques reconnues à Madagascar », Rev. Elev. Méd. vét. Pays trop. 1964, 17 (3): 477-90.

9. DESCHIENS (R.), BENEX (J.), LAMBAULT (E.), «Les conditions pratıques d'utilisation des antigènes parasitaires stabilisés dans le diagnostic immunologique des helminthiases $»$, Ann. Inst. Pasteur 1961, 101 (6): 951-64.

10. FAVATI (V.), DELLA CROCE (G.), « Diagnosis of liver fluke infestation in cattle. Value of faecal examination plus allergic test ", Amal. Fac. Med. vet., Pisa, 1964, 17: 75-86.

11. FRICK (W.), * Immunobiological detection of fascioliasis in cattle ", Arch. exp. Vet. Med. 1968, 22: 1011-13.

12. KOCH (H.W.), «Untersuchungen User die Brauchbarkeit von haemagglutination flocculation, Mikro-Agar-Präzipitatıon und des Immunofluoreszenz zum früh hzeitigen Nachweis der Fasciolose des Rindes », Inaug. Diss. Vet. Med. Fak. Freie Univ., Berlin, 1969, p. 28 (GE).

13. MATHUR (P. B.), «Versuche zur Festellung der Brauchbarkest der KBR fur die Fasciola hepaticadiagnose », Inarg. Diss. Freie Univ. Berlin, 1966, p. 29.

14. PATNAIK (B.), DAS (K.M.), «Diagnosis of fascioliasis in cattle by intra-dermal allergic test ", Cornell. Vet. 1961, 51: 113-23.

15. PAUTRIZEL (R.) et collab., * Utilisation d'un antigène distomien délipidé dans le diagnostic allergique de la distomatose à Fasciola hepatica », Rev. Immunol., Paris, 1962, $26: 167-74$.

16. SEWELL (M.M.H.), "The immunology of fascioliasis - II. Qualitative studies on the precipitin reaction ", Immunology, 1964, 7: 671-80.

17. SINCLAIR (I.J.), KENDALL (S. B.), « Precipitating antibodies to infection with Fasciola hepatica in rabbits \%, Res. vet. Sci. 1969, 10: 483-85.

18. TAILLIEZ (R.), " Isolement et étude d'un antigène spécifique de la grande douve du foie Fasciola hepatica L. », Biologie méd. 1970, 59 (3) : $183-287$

19. TOPACIO (T. M.), MARTIN (E. C.), \& The diagnostic of fascioliasis in carabaos by the use of the intra-dermal allergic test ", Philipp. J. vet. Med. 1963, 2 : 153-59.

20. VAREJCKO (J.), «An allergic, intradermal and intrapalpebral test for diagnosis of fascioliasis in cattle », Sh. vys. Sk. zeméd Brne, 1965, Ser B 13. 